\title{
Um estudo sobre as crenças de autoeficácia na resolução de tarefas matemáticas
}

\section{A study on the self-efficacy beliefs in solving mathematical tasks}

\author{
Katerine Edith Tobio-Gutiérrez \\ $\triangle$ katerine.tobio-gutierrez@unesp.br \\ UNESP - Universidade Estadual Paulista "Júlio de Mesquita Filho", Bauru, Brasil \\ Martha Mogollón-Rodríguez (iD) \\ $\triangle$ marthamogollon2018@gmail.com \\ URBE - Universidad Privada Dr. Rafael Belloso Chacín, Maracaibo, Venezuela \\ Nelson Pirola (iD) \\ $\triangle$ nelson.pirola@unesp.br \\ UNESP - Universidade Estadual Paulista "Júlio de Mesquita Filho", Bauru, Brasil
}

Recibido: 07-08-2018

Aceptado: $13-12-2018$

Publicado: $17-12-2018$

\section{Resumo}

Objetivo. Esta pesquisa tem como objetivo estudar as crenças de autoeficácia na resolução de tarefas de sequências numéricas e geométricas por alunos do Ensino Fundamental II, no município de Corozal-Sucre (Colômbia). Método. Este trabalho é de abordagem quantitativa do tipo descritivo com desenho não experimental transversal. Para a coleta de dados, foi elaborado um questionário online sobre as crenças de autoeficácia envolvendo 6 tarefas de sequências numéricas e geométricas de tipo escala Likert, aplicado a uma amostra não probabilística de 153 estudantes de sétimo, oitavo e nono ano, com idades entre os 11 aos 17 anos, quase num mesmo número entre homens e mulheres os quais, voluntariamente, quiseram participar da pesquisa. Para a análise dos dados, foi realizada uma análise estatística descritiva e probabilística. Resultados. Este estudo contribui à compreensão das crenças de autoeficácia no contexto da Educação Matemática, especialmente na resolução de tarefas sobre sequências numéricas e geométricas. Verificou-se que as crenças de autoeficácia dos alunos não estão relacionadas com às variáveis, nível educativo, gênero e idade. Além disso, o número de estudantes com crenças de autoeficácia favoráveis e desfavoráveis são similares. Conclusões. Evidenciou-se que quase a metade dos participantes possuem percepções de crenças de autoeficácia negativas na solução de tarefas de sequências numéricas e geométricas, situação preocupante, posto que estas tarefas requerem o uso de conceitos e procedimentos envolvidos na exploração de padrões, regularidades e o desenvolvimento das habilidades de identificação e generalização de padrões que é aplicado tanto no campo da matemática quanto outros campos do conhecimento.

Palavras claves: Crenças de autoeficácia, sequência, generalização de padrões, pensamento algébrico, Ensino Fundamental. 
Tobio-Gutiérrez et al - Um estudo sobre as crenças de autoeficácia na resolução de tarefas matemáticas

\section{Abstract}

Objective. This research aims to study the self-efficacy beliefs in the resolution of tasks of numerical and geometric sequences by Middle School students, in the municipality of Corozal-Sucre (Colombia). Method. This work is a quantitative approach of descriptive type with transversal non-experimental design. For data collection, an online questionnaire was elaborated on self-efficacy beliefs involving 6 tasks of numerical and geometric sequences of Likert scale type, applied to a non-probabilistic sample of 153 students of seventh, eighth and ninth grade, aged 11 to 17 years, almost in the same number of men and women who voluntarily wanted to participate in the research. For data analysis, a descriptive and probabilistic statistical analysis was performed. Results. This study contributes to the understanding of self-efficacy beliefs in the context of mathematical education, especially in the resolution of tasks on numerical and geometric sequences. It was verified that the students' self-efficacy beliefs are not related to the variables, educational level, gender and age. In addition, the number of students with favorable and unfavorable self-efficacy beliefs are similar. Conclusions. It was evidenced that almost half of the participants have perceptions negative self-efficacy beliefs in the solution of tasks of numerical and geometric sequences, a worrying situation, since these tasks require the use of concepts and procedures involved in the exploitation of patterns, regularities and the development of the skills of identification and generalization of patterns that is applied both in the field of mathematics and other fields of knowledge.

Keywords: Self-efficacy beliefs, sequence, generalization of patterns, algebraic thinking, Middle School.

\section{Introdução}

A questão da afetividade relacionada ao processo de ensino e aprendizagem da matemática aparece de forma reduzida na literatura. Quando se pesquisa as crenças de autoeficácia relacionadas à aprendizagem ou ao ensino de matemática, esses trabalhos também resultam ser escassos, conforme mostra a pesquisa de Sander (2018).

O estudo sobre a autoeficácia têm suas raízes na teoria social cognitiva, sendo Bandura (1997) seu principal representante. De acordo com Bandura (1986) o conceito de autoeficácia é considerado como o julgamento próprio das habilidades, capacidades, conhecimento, inteligência, etc., que cada indivíduo possui e usa na hora de realizar as ações necessárias para alcançar um certo grau de desempenho em um domínio específico, fazendo referência ás conviçcões pessoais em relação com suas próprias habilidades ao realizar uma determinada tarefa e o grau de qualidade da execução desta.

A crença de autoeficácia, no campo da Matemática, tem se tornado em um dos principais fatores relacionados à aprendizagem dessa disciplina, conforme destaca Rodrigues (2016). De acordo com Bandura (1994, 1997), as crenças de autoeficácia podem ser definidas como as crenças que o aluno possui em sua própria capacidade de organizar e executar cursos e ações necessárias para produzir certas conquistas relativas aos aspectos intelectuais e de aprendizagem. Quando o aluno está diante de uma tarefa 
matemática, ele pode se sentir mais ou menos confiante em sua capacidade para solucioná-la. As crenças de autoeficácia em Matemática são desenvolvidas ao longo da escolaridade e tem forte influência das experiências que os alunos tiveram em relação a essa disciplina. Por exemplo: se o aluno passou por diversas situações de fracasso ao tentar resolver tarefas matemáticas, é provável que passe a não acreditar em sua própria capacidade para resolver essas tarefas.

Tendo em mente que não necessariamente as capacidades consideradas estão presentes no sujeito, trata se de que o sujeito acredita que possui aquelas habilidades, além disso, ter a disposição para contemplar as demandas de uma determinada tarefa que precisa ser realizada (Bzuneck, 2001), as crenças de autoeficácia assumem um papel preditivo e de mediação com respeito à motivação, bem-estar, disposição e a resiliência do indivíduo, dando origem a ações para o desenvolvimento de um domínio específico (Bandura, 1997; Caprara et al., 2006).

Bandura (1986, 1994,1997) em seus estudos identificou quatro principais fontes que constituem as crenças de autoeficácia, quais sejam: A experiência direta, representadas pelo desenvolvimento da tarefa a partir da mensuração dos efeitos e interpretações das ações realizadas; a experiência vicária, relacionadas à observação de modelos ou imitação no desenvolvimento de tarefas desempenhadas por outros; a persuasão verbal, referindo-se às formas de orientação, avaliação, sugestões, pontos de vista e críticas; finalmente, os estados somáticos e emocionais, que resultam das ativações psicofisiológicas relativas à organização e execução de uma tarefa, como ansiedade, fadiga, estresse, etc.

As crenças de autoeficácia têm sido objeto de interesse em pesquisas em várias áreas do conhecimento e contextos, como saúde, psicologia, administração, esportes, incluindo o ensino de Matemática, envolvendo diferentes conteúdos matemáticos. Uma revisão da literatura mostra que esse tema, ainda é pouco conhecido e difundido no contexto educacional colombiano. No contexto educacional brasileiro e latino-americano encontramos alguns estudos que abordam a relação do constructo autoeficácia com; os objetivos no campo da aprendizagem (Patrício, 2012); atitudes (Dobarro, 2007); o desempenho na resolução de situações de problemas (Brito e Souza, 2015; Vargas et al., 2015, Maldonado, 2016, Sander, 2018); as atribuições de sucesso e fracasso (Morais, 2016); autoconceito (Souza e Brito, 2008) e o ensino de álgebra na formação de professores (Pinheiro, 2018).

O estudo desta temática se deve ao interesse em conhecer as percepções das crenças de autoeficácia matemática presentes nos participantes da pesquisa. Ainda, devido à importância do diálogo do professor-aluno-conhecimento no processo de ensino e aprendizagem do ponto de vista educativo e científico, posto que o baixo desempenho dos estudantes muitas vezes é atribuído, por parte dos docentes, por outros fatores distinto a aspecto afetivos e atitudinais (Dabarro, 2007).

Por outro lado, deve-se notar que, dentro dos Estandares Básicos de Competências em Matemática do Ministério da Educação Nacional da Colômbia (MEN, 2006), é abordada a generalização de padrões em tarefas de sequências ligadas ao pensamento variacional e aos sistemas algébricos e analíticos, descrevendo que o estudo de padrões está intimamente relacionado com o desenvolvimento do pensamento variacional, permitindo 
o reconhecimento de regularidades e a detecção de critérios, unidades de repetição ou regras que governam a formação de uma sequência de acordo com um padrão existente e a organização dos elementos que as compõem. Da mesma forma, o processo de reconhecer as diferenças e semelhanças entre os termos de uma sequência desenvolve a capacidade de identificar padrões, continuar a reprodução de uma sequência, ratificar ou refutar as hipóteses iniciais e o processo de generalização. Assim, o MEN (2006) recomenda a realização de tarefas de análise dos fenômenos variacionais e de generalização de padrões nos níveis de Ensino Fundamental I, esta é considerada como uma maneira adequada para o desenvolvimento de uma aprendizagem significativa e abrangente dos sistemas algébricos, bem como a sua gestão simbólica antes de atingir o sétimo e oitavo anos do Ensino Fundamental II.

Por esta razão, o desenvolvimento de solução de tarefas de sequências no processo de ensino e aprendizagem é essencial, devido à contribuição que dá para a construção de conceitos e procedimentos relacionados com as expressões algébricas que possibilitam a criação de uma regra para encontrar os termos seguintes a partir dos termos conhecidos, por meio do reconhecimento e generalização do padrão correspondente à situação dada, considerando que nos níveis de Ensino Fundamental II o sistema algébrico está diretamente relacionado às variações e expresso em diferentes tipos de representações que fazem parte do pensamento algébrico (Zapatera, 2018; MEN, 2006).

Além disso, as sequências numéricas e geométrica fazem parte do conteúdo temático de estudo no campo da Educação Matemática para investigar o raciocínio indutivo (Cañadas, 2007, Álvarez, 2012), pensamento lógico matemático (Morales, 2013), as estratégias para generalização de padrões e sua contribuição para o desenvolvimento do pensamento algébrico em estudantes de diferentes níveis de ensino (Zapatera, 2018).

Portanto, o objetivo deste artigo é estudar as crenças de autoeficácia na solução de tarefas de sequências numéricas e geométricas de uma amostra não probabilística de 153 estudantes de sétimo, oitavo e nono ano de uma escola pública do município de CorozalSucre (Colômbia), utilizando como instrumento de coleta de dados um questionário de escala Likert das crenças de autoeficácia, que foi aplicado de maneira online, contendo tarefas de sequência numéricas e geométricas. Posteriormente, foi realizada uma análise estatística descritiva e probabilística dos dados.

Neste trabalho, se considera que as tarefas são entendidas como um segmento de atividade de classe, orientado para o desenvolvimento de um conceito matemático particular que pode conter diferentes situações Problemas relacionados ou um amplo trabalho sobre um problema complexo (Barbosa, 2010).

\section{Método}

Esta pesquisa é de abordagem quantitativa do tipo descritiva com desenho não experimental transversal, pois objetivou-se, neste trabalho, a descrever o grau de crenças de autoeficácia que os alunos do Ensino Fundamental II têm em relação à solução de tarefas de sequências numéricas e geométricas no contexto escolar, num determinado momento (Hernández Sampieri et al., 2014). Considerou-se esta perspectiva adequada, dado que pretendia-se estar mais próximo à realidade dos participantes estabelecendo um espaço e tempo para coletar os dados, sem modificar ou intervir na variável de 
estudo e no contexto natural, permitindo estudar as percepções que possuem os estudantes sobre as crenças de autoeficácia nas tarefas matemáticas, determinadas no momento que foi aplicado o instrumento de coleta de dado, segundo sua categoria, crenças favoráveis (positivas) e crenças desfavoráveis (negativa) e tendo em conta as variáveis intervenientes, como idade, gênero e nível escolar dos alunos para encontrar possíveis relações com a variável de estudo.

Tabela 1. Distribuição da frequência do gênero versus nível escolar

\begin{tabular}{|c|c|c|c|c|c|c|}
\hline & & & \multirow[b]{2}{*}{70} & \multicolumn{2}{|c|}{ Ano Escolar } & \multirow{2}{*}{ Total } \\
\hline & & & & $8^{\circ}$ & $9^{\circ}$ & \\
\hline \multirow{4}{*}{ Gênero } & \multirow{2}{*}{$M$} & Contagem & 41 & 18 & 18 & 77 \\
\hline & & $\%$ & $26,8 \%$ & $11,8 \%$ & $11,8 \%$ & $50,3 \%$ \\
\hline & \multirow{2}{*}{$\mathrm{F}$} & Contagem & 56 & 9 & 11 & 76 \\
\hline & & $\%$ & $36,6 \%$ & $5,9 \%$ & $7,2 \%$ & $49,7 \%$ \\
\hline \multicolumn{2}{|l|}{ Total } & Contagem & 97 & 27 & 29 & 153 \\
\hline \multicolumn{2}{|l|}{$\%$} & $63,4 \%$ & $17,6 \%$ & $19,0 \%$ & $100,0 \%$ & \\
\hline
\end{tabular}

Fonte: elaboração própria.

Este estudo possui uma amostra não probabilística, pois os participantes desta pesquisa são pertencentes a uma escola pública do Município de Corozal-Sucre (Colômbia), sem serem selecionados com técnicas estatísticas e sem a pretensão de estender a pesquisa a outras escolas do Município, já que, não se tem nenhum interesse em extrapolar os resultados desta pesquisa (Hernández Sampieri et al., 2014). A amostra está constituída por 153 estudantes, em que 50,3\% são do sexo masculino (M) e 49,7\% são do sexo feminino ( $F$ ). Além disso, 63,4\% correspondem a estudantes de sétimo, 17,6\% ao oitavo e $19 \%$ ao nono ano, com idades entre 11 a 17 anos, da escola Liceo Carmelo Percy Vergara.

Para a coleta dos dados, foi elaborado um questionário de crença de autoeficácia, do tipo escala Likert, contendo 6 tarefas de sequências numéricas e geométricas, com pontuação que variava de 1 a 5, que foi embasada em outras escalas de crenças de autoeficácia (Dobarro, 2007; Sander, 2018). Também foram elaboradas tarefas de sequências numéricas e geométricas (Cañadas, 2007; Zapatera, 2018; Alvarez, 2012). Este questionário apresenta cinco tarefas que possuem uma pergunta (Figura 1) e a última tarefa contém 9 questões (Figura 2). A aplicação do questionário foi realizada de forma digital na sala de aula de informática da escola, com uma duração média de 15 minutos.

Em geral, essas tarefas propõem encontrar elementos próximos (tarefas 1, 4, 6a, 6B e 6F) e distantes (tarefas 2, 3, 6C e 6G) de uma determinada sequência, assim como, explicar com as suas próprias palavras a relação direta (tarefa 6D) e inversa (6h) das variáveis presentes em cada sequência, além de expressar descritiva e matematicamente a relação direta (tarefa 5, 6E e 6H) e inversa (tarefa 6I) das variáveis na tarefa. 
Tobio-Gutiérrez et al - Um estudo sobre as crenças de autoeficácia na resolução de tarefas matemáticas

\begin{tabular}{|c|c|c|c|c|c|c|}
\hline $\mathrm{N}^{\circ}$ & Tarea & & & & & \\
\hline 1 & $\begin{array}{l}\text { Se tiene la siguiente secuencia de números: } 3,7,13,21 \ldots \\
\text { Escribe los cuatro números siguientes de la secuencia, justificando } \\
\text { tu respuesta. }\end{array}$ & 1 & 2 & 3 & 4 & 5 \\
\hline 2 & $\begin{array}{l}\text { Se tiene la siguiente secuencia de números: } 1,4,7,10, \ldots \\
\text { Escribe el número que estará en la posición } 213 \text {, justificando tu } \\
\text { respuesta. }\end{array}$ & 1 & 2 & 3 & 4 & 5 \\
\hline 3 & $\begin{array}{l}\text { Imagina que tienes unas baldosas cuadradas blancas y otras baldosas } \\
\text { cuadradas grises son del mismo tamaño. Hacemos una fila con las } \\
\text { baldosas blancas: } \begin{array}{|l|l|l|l|l|l|l|} & & & & \\
\end{array} \\
\text { Rodeamos las baldosas blancas con baldosas grises, tal y como } \\
\text { muestra el dibujo: }\end{array}$ & 1 & 2 & 3 & 4 & 5 \\
\hline 4 & $\begin{array}{l}\text { Números triangulares: Se llama número triangular un número que } \\
\text { puede ser representado por medio de un triángulo como se observa a } \\
\text { seguir: } \\
\text { ¿Cuántos puntos debe tener la figura de la posición } 10 \text { ? }\end{array}$ & 1 & 2 & 3 & 4 & 5 \\
\hline 5 & 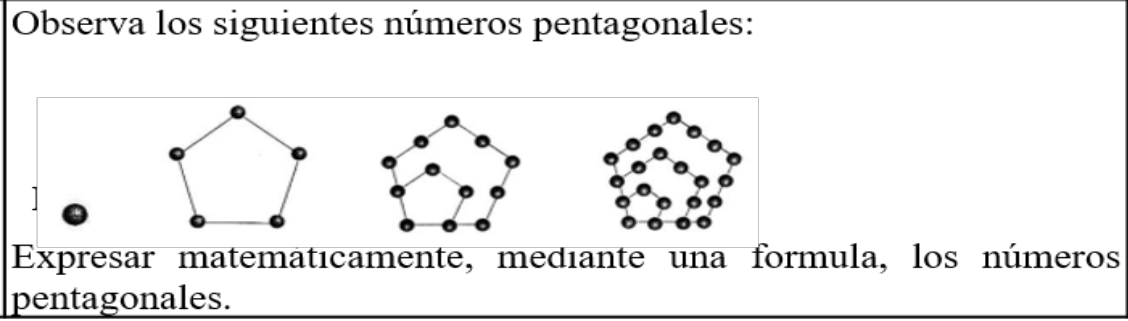 & 1 & & 3 & 4 & \\
\hline
\end{tabular}

Figura 1. Tarefas apresentadas no questionário de escala de crenças de autoeficácia (parte 1). Fonte: Elaboração própria. (link: https://goo.gl/forms/xqCKfJdt8AXzgyc73).

Portanto, realizou-se o seguinte procedimento:

Fase I: Foram selecionados os estudantes que, voluntariamente, quiseram participar da pesquisa e tinham o termo de consentimento livre e esclarecido assinalado pelos pais ou pessoas responsáveis.

Fase II: Aplicação do questionário aos participantes que tinham o termo de consentimento livre e esclarecido assinalado nesse momento.

Fase III: Análise dos dados. Primeiramente, foi analisada a confiabilidade do questionário, calculando-se o coeficiente alfa de Cronbach $(0,704)$, que é considerado aceitável para este tipo de pesquisa (Hernández Sampieri et al., 2014). Posteriormente, os dados foram analisados mediante uma análise descritiva, de igual maneira, uma análise probabilística para relacionar a variável de interesse do estudo com as variáveis intervenientes, utilizandose o software livre $R$-Studio e o pacote Rcommander (Fox J, Bouchet-Valat M., 2017). 


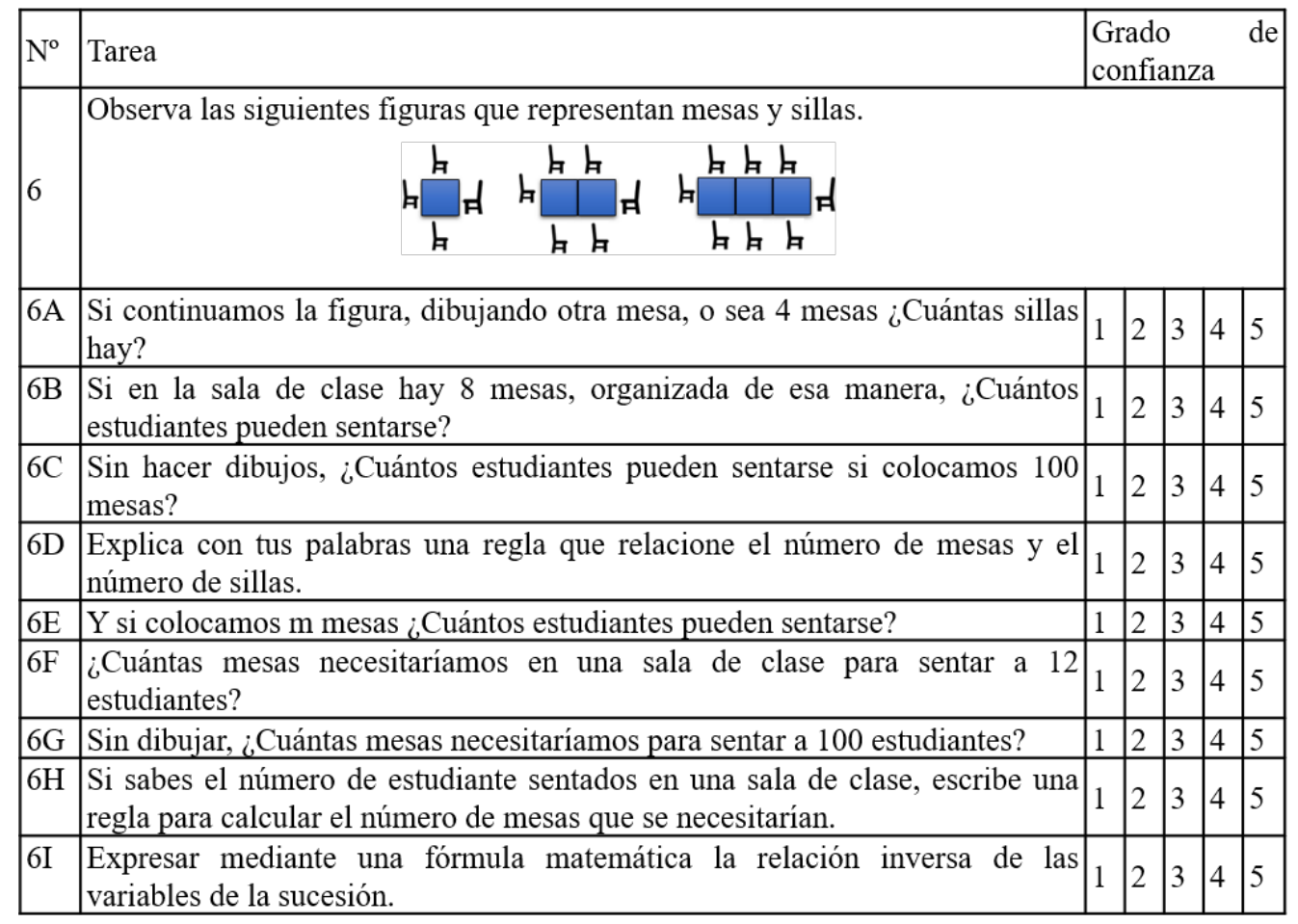

Figura 2. Tarefas apresentadas no questionário de crença de autoeficácia (parte 2). Fuente: elaboração própria (link: https://goo.gl/forms/xqCKfJdt8AXzgyc73).

\section{Resultados}

Para a análise do grau de crença de autoeficácia dos 153 estudantes na solução das tarefas de sequências numéricas y geométricas, foi feita a soma dos pontos atingidos por cada estudante os quais variaram entre 14 e 70 pontos, isto porque o questionário tinha 14 itens distribuídos nas 6 tarefas e a escala variava de 1 a 5 pontos por cada tarefa. Em seguida, com os resultados dos pontos, calculou-se a média aritmética $(48,46)$ e o desvio padrão $(7,54)$. Aqueles estudantes, cujo escore foi acima da média, demonstraram crenças positivas de autoeficácia na solução das tarefas de sequências numéricas e geométricas e no caso contrário, elas são consideradas como crenças negativas (Brito e Souza, 2008, 2015; Sander, 2018).

Portanto, descreveremos os resultados do grau de autoeficácia que os alunos consideram em cada item $\left(\mathrm{N}^{\circ}\right)$ e, posteriormente, de forma geral. Para a análise de cada item é tomado que todos os alunos que têm valores acima do valor da média obtida na escala de autoeficácia têm crenças favoráveis de autoeficácia $(A E+)$ e abaixo e igual à média de cada item, têm crenças desfavoráveis (AE-), como é mostrado na Tabela 2.

Portanto, se analisarmos os escores do grau de autoeficácia de cada tarefa (Tabela 2), observa-se que os alunos têm crenças de autoeficácia positiva nas tarefas que se propõem a encontrar elementos próximos de uma relação direta (tarefa 6B) e inversa (6F), das variáveis envolvidas na sequência. Dessa forma, não existe uma diferença significativa entre o número de alunos que possuem uma crença de autoeficácia favorável e aqueles que têm uma crença desfavorável na realização de tarefas de sequências numéricas 
e geométricas. Em relação às crenças negativas, destacam-se aquelas tarefas que propõem encontrar elementos distantes (tarefas 2 e 3) de uma determinada sequência, bem como, para explicar com as suas próprias palavras a relação direta (tarefa 6D) e inversa (6h) e expressar de forma descritiva e matematicamente a relação direta (tarefa $5,6 e$ e $6 \mathrm{~h}$ ) e inversa (tarefa $6 \mathrm{I}$ ) das variáveis da sequência.

Tabela 2. Distribuição de frequências das categorias de crenças de autoeficácia segundo o gênero.

\begin{tabular}{|c|c|c|c|c|c|c|}
\hline \multirow{2}{*}{ No } & \multirow{2}{*}{ Media } & \multirow{2}{*}{ DP } & \multicolumn{2}{|c|}{ AE+ } & \multicolumn{2}{|c|}{ AE- } \\
\hline & & & $\mathbf{F}$ & $\mathbf{M}$ & $\mathbf{F}$ & M \\
\hline 1 & 3,47 & 1,07 & $38(24,8 \%)$ & $34(22,2 \%)$ & $38(24,8 \%)$ & $43(28,1 \%)$ \\
\hline 2 & 3,43 & 0,99 & $37(24,2 \%)$ & $39(25,5 \%)$ & $59(51,8 \%)$ & $44(28,8 \%)$ \\
\hline 3 & 3,28 & 1,18 & $33(21,6 \%)$ & $36(23,5 \%)$ & $43(28,1 \%)$ & $41(26,8 \%)$ \\
\hline 4 & 3,47 & 1,09 & $30(19,6 \%)$ & $46(30,1 \%)$ & $46(30,1 \%)$ & $31(20,3 \%)$ \\
\hline 5 & 3,12 & 1,02 & $24(15,7 \%)$ & $25(16,3 \%)$ & $52(34,0 \%)$ & $52(34,0 \%)$ \\
\hline $6 \mathrm{~A}$ & 4,16 & 0,99 & $39(25,5 \%)$ & $38(24,8 \%)$ & $37(24,2 \%)$ & $39(25,5 \%)$ \\
\hline $6 B$ & 3,93 & 1,03 & $56(36,6 \%)$ & $54(35,3 \%)$ & $20(13,1 \%)$ & $23(15,0 \%)$ \\
\hline $6 C$ & 3,49 & 1,13 & $39(25,5 \%)$ & $37(24,2 \%)$ & $37(24,2 \%)$ & $40(26,1 \%)$ \\
\hline $6 \mathrm{D}$ & 3,34 & 1,05 & $30(19,6 \%)$ & $35(22,9 \%)$ & $46(30,1 \%)$ & $42(27,5 \%)$ \\
\hline $6 \mathrm{E}$ & 3,04 & 1,28 & $23(15,0 \%)$ & $33(21,6 \%)$ & $53(34,6 \%)$ & $44(28,8 \%)$ \\
\hline $6 \mathrm{~F}$ & 3,86 & 1,06 & $46(30,1 \%)$ & $53(34,6 \%)$ & $30(19,6 \%)$ & $24(15,7 \%)$ \\
\hline $6 G$ & 3,5 & 1,18 & $38(24,8 \%)$ & $39(25,5 \%)$ & $38(24,8 \%)$ & $38(24,8 \%)$ \\
\hline $6 \mathrm{H}$ & 3,24 & 1,16 & $28(18,3 \%)$ & $37(24,2 \%)$ & $48(31,4 \%)$ & $40(26,1 \%)$ \\
\hline $6 \mathrm{I}$ & 3,07 & 1,17 & $28(18,3 \%)$ & $31(20,3 \%)$ & $48(31,4 \%)$ & $46(30,1 \%)$ \\
\hline
\end{tabular}

Fuente: elaboração própria.

Da mesma forma, podemos enfatizar que o número de estudantes do gênero masculino apresenta maiores crenças de autoeficácia favoráveis em relação às alunas nas tarefas que propõem encontrar elementos próximos (tarefa 4 e 6F) e distantes ( Tarefas 6G). Além disso, as estudantes do sexo feminino têm maior porcentagem de crenças autoeficácia em relação à tarefa $6 \mathrm{~A}$, ao encontrar elementos próximos. Considerando a análise dos itens do questionário, podemos afirmar que os alunos apresentam crenças desfavoráveis de autoeficácia na maioria das tarefas de sequências numéricas e geométricas.

Na análise estatística descritiva geral dos dados, obteve-se que 81 (52,9\%) participantes apresentam tendência a crenças de autoeficácia positiva, dos quais $53(34,6 \%)$ estudantes são de sétimo ano, $13(8,5 \%)$ são de oitavo e $15(9,8 \%)$ do nono ano, enquanto 72 $(47,1 \%)$ participantes apresentam tendências negativas, em que $44(28,8 \%), 14(9,2 \%)$ e $14(9,2 \%)$ são alunos do sétimo, oitavo e nono ano, respectivamente.

Embora o número de alunos com crenças de autoeficácia positiva seja maior que as crenças de autoeficácia negativa, podemos enfatizar que o número de alunos nos diferentes níveis de ensino não apresenta um aumento progressivo em relação às crenças de autoeficácia positiva, já que $48,15 \%$ dos alunos de oitavo ano têm crenças de autoeficácia favoráveis em comparação com os alunos do sétimo e nono ano que possuem mais do $50 \%$, tendo o sétimo ano o maior número de alunos com crenças positivas de autoeficácia com 54,64\%. Além disso, constatamos que o número de alunos com crenças de autoeficácia desfavoráveis em cada nível de escolaridade aumenta, em que o $51,85 \%$ dos alunos do oitavo ano tem crenças de autoeficácia negativa. 
Por conseguinte, se realiza a análise probabilística usando o teste de hipótese Quiquadrado entre as categorias da variável crenças de autoeficácia, classificada como autoeficácia positiva e negativa e as variáveis intervenientes como nível escolar, gênero e idade, a fim de encontrar relações entre variáveis. Foram encontrados os resultados: crenças de autoeficácia com nível escolar $(x 2=0,378, p>0,828)$, gênero $(x 2=$ $1,098, p>0,295)$ e idade $(x 2=1,074, p>0,584)$. Portanto, não se encontrou relação estatisticamente significativas entre a variável de estudo e as variáveis interveniente.

\section{Discussão}

Segundo os resultados obtidos, ressalta-se que a sexta tarefa foi aplicada em um estudo sobre a generalização dos padrões por Zapatera (2018) com alunos de terceiro ao sexto ano do Ensino Fundamental I, afirmando que a maioria dos alunos que participaram do estudo conseguiu encontrar os elementos próximos da sequência. Foram encontradas evidências de que os alunos apresentaram dificuldades em reverter o pensamento, ou seja, encontrar a relação inversa das variáveis da sequência que Ihes permitia determinar os termos próximos e distantes, e expressar verbalmente a regra geral de forma algébrica. Sendo assim, podemos afirmar que os participantes deste estudo têm crenças de autoeficácia semelhantes ao desempenho de alunos do Ensino Fundamental I. Lembrando que este estudo não aborda o desempenho dos participantes na solução de tarefas de sequências numéricas e geométricas, mas sim suas crenças de autoeficácia, ou seja, os alunos não resolveram as tarefas de sequência numéricas e geométricas. Pode-se dizer que esta afirmação é significativamente preocupante, uma vez que o nosso trabalho foi realizado com alunos de sétimo, oitavo e nono ano do Ensino Fundamental II e, portanto, as estruturas mentais em matemática destes estudantes devem ser superiores aos dos alunos do Ensino Fundamental I.

O grau das crenças de autoeficácia negativa por parte dos alunos na resolução de tarefas de sequências numéricas e geométricas pode estar associado à falta de exploração de padrões, regularidades e ao processo de generalização em diferentes situações contextualizadas em sala de aula que proporcionam diferentes formas de resolver problemas de identificação e generalização de padrões (Zapatera, 2018); 0 desenvolvimento e a compreensão do pensamento indutivo (Álvarez, 2012; Cañadas, 2007); e sua articulação com outros conteúdos e áreas matemáticas do conhecimento nos diferentes níveis de ensino (Álvarez, 2012). Por isso, recomenda-se desenvolver estudos posteriores com relação às crenças de autoeficácia e o desempenho nas tarefas de sequências numéricas e geométricas que abordem esta problemática.

Os resultados encontrados na análise probabilística com relação a variável de estudo e as variáveis intervenientes não estão de acordo com os resultados obtidos por Brito e Souza (2015), visto que seus resultados demonstram significativamente que as meninas têm uma crença de autoeficácia mais forte que os meninos. Mas, estão de acordo com os trabalhos de Pinheiro (2018) e Dobarro (2007), posto que não foram identificadas diferenças significativas entre as crenças de autoeficácia, quando foram agrupados por gênero e nível escolar.

\section{Conclusões}

De acordo com a análise da literatura e da revisão bibliográfica, evidenciou-se que não existem muitos estudos sobre crenças de autoeficácia, relacionadas aos conceitos 
e procedimentos envolvidos nas sequências numéricas e geométricas. Deste modo, acreditamos que a temática, crenças de autoeficácia, merece ser pesquisada com maior aprofundamento, visto que possui influências na aprendizagem dos alunos e no desempenho escolar. Como resultado deste estudo, as crenças de autoeficácia positivas e negativas não estão relacionadas às variáveis como o nível escolar, gênero e idade dos estudantes.

Este estudo pretende contribuir na compreensão das crenças de autoeficácia no contexto da Educação Matemática, especialmente na resolução de tarefas sobre sequências numéricas e geométricas, destacando-se que quase a metade dos participantes possui percepções de crenças de autoeficácia negativas, situação preocupante, posto que estas tarefas requerem mobilização de conceitos e procedimentos envolvidos na exploração de padrões, regularidades e o desenvolvimento das habilidades de identificação e generalização de padrões, sendo a generalização um processo cognitivo importante da atividade matemática.

Além disso, para futuras pesquisas sobre o assunto, recomenda-se aplicar a mais alunos de distintos níveis de escolaridade que permite obter uma maior consistência interna para a avaliação de crenças de autoeficácia em soluções de sequências numéricas e geométricas, embora o cálculo do coeficiente Alfa de Cronbach $(0,704)$ seja considerado estatisticamente aceitável. Também é desejável que haja um aprofundamento do desempenho na exploração de padrões, regularidades e o desenvolvimento das habilidades de identificação e generalização de padrões nos estudantes nos diferentes níveis de escolaridade posto que são muito importantes no processo cognitivo dos alunos e na solução de situações problemas, tanto no campo matemático quanto em outros campos do conhecimento mediante o desenvolvimento de estudo que abordem esta problemática.

\section{Referências}

Álvarez, L. M. R. (2012). Patrones y Regularidades Numéricas: Razonamiento Inductivo. (Tese de mestrado). Universidade Nacional de Colombia, Bogotá. Disponível em http://bdigital.unal.edu.co/39503/1/1186541.2014.pdf

Bandura, A. (1986). Social foundations of thought and action. Englewood Cliffs, NJ.

Bandura, A. (1997). Self-efficacy: The exercise of control. New York: W. H. Freeman and Company.

Bandura, A. (1994). Self-efficacy. In Ramachaudran, V. S. (Ed.), Encyclopedia of human behavior. 4, pp. 71-81. New York: Academic Press, 1994. (Reprinted in H. Friedman [Ed.], Encyclopedia of mental health. San Diego: Academic Press, 1998).

Barbosa, A. (2010). A resolução de problemas que envolvem a generalização de padrões em contextos visuais: Um estudo longitudinal com alunos do $2 .{ }^{\circ}$ ciclo do ensino básico. (Tese de doutorado). Universidade do Minho, Braga, Portugal. Disponível em https://core.ac.uk/download/pdf/55611252.pdf 
Brito, M. R F. \& Souza, L. F. N. I. (2015). Autoeficácia na solução de problemas matemáticos e variáveis relacionadas. Temas em Psicologia, 23(1). DOI: 10.9788/TP2015.1-03

Bzuneck, J. A. (2001). As crenças de auto-eficácia e o seu papel na motivação do aluno. Em E. Boruchovitch \& J.A. Bzuneck (Org.) A Motivação do Aluno: Contribuições da Psicologia Contemporânea (pp. 116-133). Petrópolis: Editora Vozes. Disponível em https://www.uky.edu/ eushe2/Pajares/Bzuneck2.pdf

Cañadas, M. (2007). Descripción y caracterización del razonamiento inductivo utilizado por estudiantes de educación secundaria al resolver tareas relacionadas con sucesiones lineales y cuadráticas. (Tese de doutorado). Universidade de Granada, Granada, Espanha. Disponível em http://funes.uniandes.edu.co/282/1/CannadasM07-2850.pdf

Caprara, G., Barbaranelli, C., Steca, P., \& Malone, P. (2006). Teachers' self-efficacy beliefs as determinants of job satisfaction and students' academic achievement: A study at the school level. Journal of School Psychology, 44(6), 473-490. Disponível em https://www.redalyc.org/pdf/167/16746507020.pdf

Dobarro, V.R. (2007). Solução de problemas e tipos de mente matemática: relações com as atitudes e crenças de autoeficácia. (Tese de Doutorado). Universidade Estadual de Campinas, Campinas, Brasil. Disponível em http://repositorio.unicamp. br/bitstream/REPOSIP/319211/1/Dobarro VivianeRezi D.pdf

Fox J, Bouchet-Valat M (2017). Getting Started with the R Commander. Version 2.4-0. Disponível em http://socserv.socsci.mcmaster.ca/jfox/Misc/Rcmdr/Getting-Startedwith-the-Rcmdr.pdf.

Hernández Sampieri, R., Fernández Collado, C., \& Baptista Lucio, P. (2014). Metodología de la investigación. Sexta Edición. Editorial Mc Graw Hill. México.

Maldonado, I., \& Isabel, C. (2016). La autoeficacia en el rendimiento académico y la capacidad de resolución de problemas matemáticos en estudiantes de cuarto grado de secundaria de un colegio estatal de San Juan de Lurigancho. (Tese de mestrado). Universidade Ricardo Palma, Lima, Peru. Disponível em http://repositorio.urp.edu. pe/handle/urp/992

Ministerio de Educación Nacional (MEN). República de Colombia. (2006). Estándares Básicos de Competencias en Matemáticas. Bogotá. Disponível em https://www. mineducacion.gov.co/1621/articles-340021 recurso 1.pdf

Morais, J. A. R. S (2016). Atribuição de sucesso e fracasso e as crenças de autoeficácia Matemática: Um estudo com alunos do Ensino Médio. (Tese de mestrado). Universidade Estadual Paulista "Júlio de Mesquita Filho", Bauru, Brasil.

Morales, R. (2013). Pensamiento lógico matemático en alumnos de 6-7 años en tareas de seriaciones, (Trabalho de fim de curso do Master). Universidade de Granada, Granada, Espanha. Disponível em http://funes.uniandes.edu.co/2131/ 
Patrício, A. S. A. (2012). Crenças de autoeficácia e objetivos: um estudo exploratório. (Tese de mestrado). Universidade de Lisboa, Lisboa, Portugal. Disponível em http:// repositorio.ul.pt/bitstream/10451/7822/1/ulfpie043043 tm.pdf

Pinheiro, A. C. (2018). O ensino de álgebra e a crença de autoeficácia docente no desenvolvimento do pensamento algébrico. (Tese de mestrado). Universidade Estadual Paulista "Júlio de Mesquita Filho", Bauru, Brasil. Disponível em https:// repositorio.unesp.br/handle/11449/154898

Rodrigues, C. S. (2015). Crenças de autoeficácia matemática na Educação de Jovens e Adultos: um estudo com alunos de Ensino Médio de Divinópolis. (Tese de mestrado). Universidade Federal de Ouro Preto, Ouro Preto, Brasil. Disponível em http://www. repositorio.ufop.br/handle/123456789/5692

Sander, G. P. (2018). Um estudo sobre a relação entre a crença de autoeficácia na resolução de tarefas numéricas e o sentido de número de alunos do Ciclo de Alfabetização. (Tese de doutorado). Universidade Estadual Paulista "Júlio de Mesquita Filho", Bauru, Brasil. Disponível em https://repositorio.unesp.br/handle/11449/154814

Souza, L. F. N. I., \& Brito, M. R. F. (2008). Crenças de auto-eficácia, autoconceito e desempenho em matemática. Estudos de Psicologia (Campinas), 25(2), 193-201. Disponível em http://www.scielo.br/pdf/estpsi/v25n2/a04v25n2.pdf

Vargas, O. L., \& Vallejo, N. G. V. (2015). Diferencias individuales en el desarrollo de la autoeficacia y el logro académico: El efecto de un andamiaje computacional. Acta colombiana de psicología, 15(2), 29-41. Disponível em https://editorial.ucatolica. edu.co/ojsucatolica/revistas ucatolica/index.php/acta-colombiana-psicologia/ article/view/264

Zapatera Llinares, A. (2018). Cómo alumnos de educación primaria resuelven problemas de generalización de patrones. una trayectoria de aprendizaje. Revista Latinoamericana de Investigación en Matemática Educativa, 21(1),87-114. DOI: 10.12802/relime.18.2114 